\title{
Epidemiología y manejo de fracturas de pelvis en el servicio de Traumatología y Ortopedia del Centenario Hospital Miguel Hidalgo
}

Martínez-Pérez Ricardo*, Soto-Juárez Ignacio**

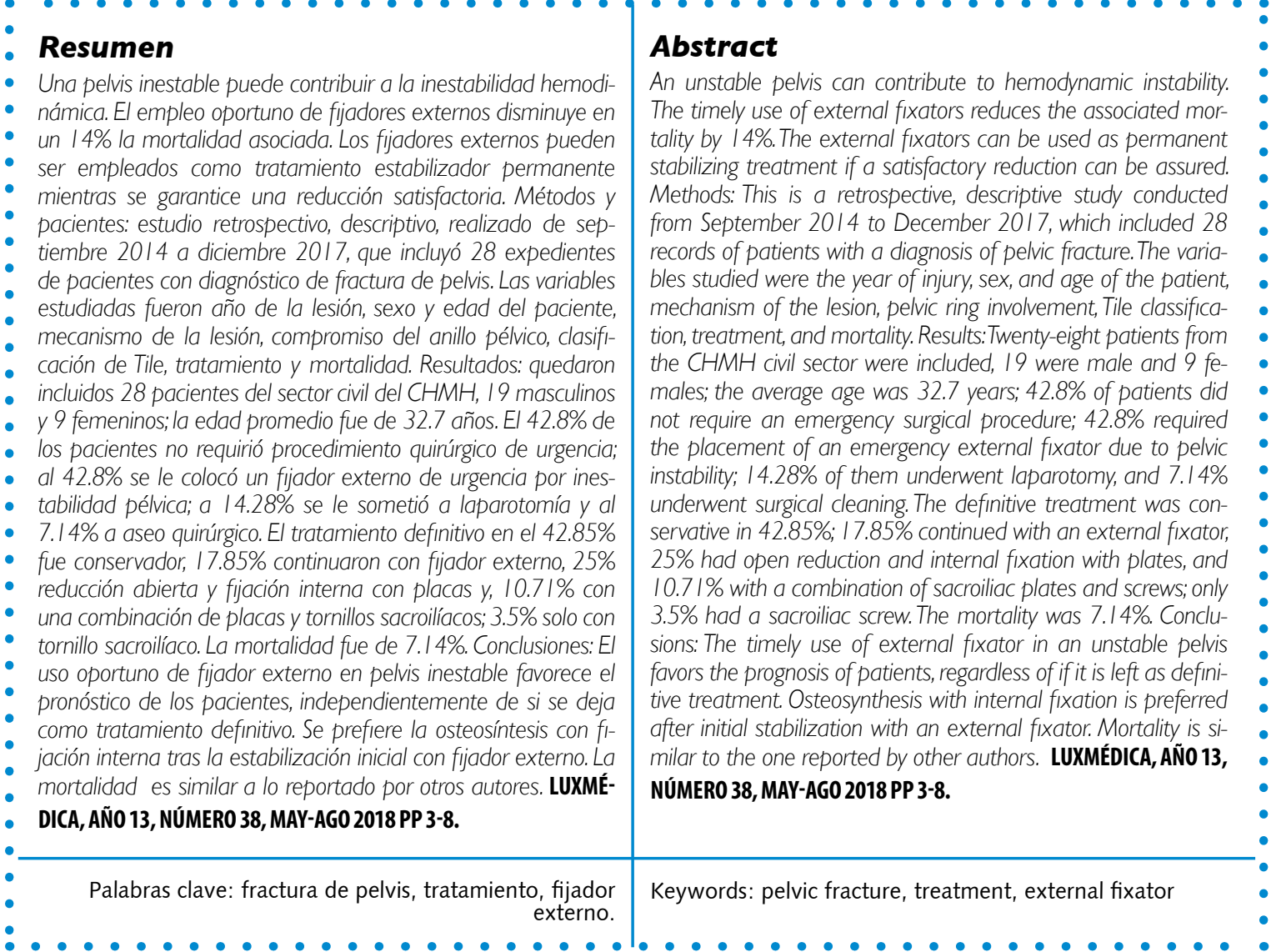

** Residente de segundo año de la especialidad de Traumatología y Ortopedia, Centenario Hospital Miguel Hidalgo, Aguascalientes, Ags, México. correo electrónico: open_shell@hotmail.com

** Médico Adscrito Titular del Módulo de Pelvis y Acetábulo del Servicio de Traumatología y Ortopedia, Centenario Hospital Miguel Hidalgo, Aguascalientes, Ags, México.

Fecha de recibido: 5 de febrero 2018

Fecha de aceptación: 5 de marzo de 2018

Correspondencia: Dr Ignacio Soto Juárez, Departamento de Ortopedia y Traumatología del Centenario Hospital Miguel Hidalgo. Calle Galeana Sur 465 Colonia Obraje, código postal 20000 Aguascalientes, Ags. México teléfono 01449994 6720, correo electrónico ignaciosotojuarez@hotmail.com 


\section{Introducción}

El manejo oportuno y adecuado de las fracturas de pelvis inestables constituye una urgencia ortopédica en todos los servicios de traumatología, por los altos índices de mortalidad y la frecuencia con la que éstas se presentan, ya que es bien conocida su asociación a accidentes de tránsito. Tan sólo en 2015, se registraron un total de 378, 232 en México, de los cuales 19.4\% resultaron con víctimas heridas, $79.5 \%$ solo con daños materiales y $1.1 \%$ con al menos una víctima mortal. Así mismo, en el estado de Aguascalientes se registraron 74 muertos, con un índice de 5.6 muertos por cada 100,000 habitantes. ${ }^{1,2}$

Las fracturas pélvicas comprenden un amplio espectro de lesiones que van de fracturas en hueso osteoporótico por mecanismos de baja energía a fracturas por alta energía con disrupción del anillo pélvico, ${ }^{3}$ la cual permite el escape de grandes cantidades de sangre a las correderas y al espacio retroperitoneal. Una pelvis inestable puede contribuir a la inestabilidad hemodinámica secundaria a lesión esquelética, así como vascular, visceral, ya que sólo el 13\% de las fracturas pélvicas son lesiones óseas aisladas. ${ }^{4}$

En 1980, Marvin Tile presentó una clasificación basada en los criterios de estabilidad pélvica, resultando en tres grupos: $A, B$ y $C$. Para su comprensión, las fracturas A no comprometen la integridad del anillo pelviano posterior y se consideran fracturas estables, con excepcionales indicaciones de tratamiento quirúrgico. En el grupo $B$, existe una interrupción parcial del anillo pelviano posterior, por lo tanto, son parcialmente inestables, caracterizadas por inestabilidad rotacional. Por último, en el grupo $\mathrm{C}$, existe interrupción completa de todas las estructuras óseas y ligamentarias del anillo posterior con una inestabilidad rotacional y vertical. Todas son inestables y requieren de estabilización quirúrgica, ${ }^{5}$ ya sea con reducción abierta y fijación interna; el uso de clamps-C, así como el empleo de fijadores externos, de utilizarse oportunamente disminuyen en un $14 \%$ la mortalidad asociada. ${ }^{6}$

Se han descrito, entre otras, dos técnicas básicas para la colocación de pines de fijadores externos pélvicos: supra-acetabular y en las crestas iliacas; las técnicas de fijación tienen por objetivo reestablecer la integridad de los componentes anterior y posterior del anillo pélvico. ${ }^{7}$ Las técnicas más comunes para la fijación del anillo pélvico posterior incluyen la placa sacroilíaca anterior (PSA) y la fijación interna con tornillos sacroilíacos percutáneos (TSP) ${ }^{8}$ ésta última resulta en una técnica menos invasiva, con menor sangrado, menos dolor y una recuperación más rápida en comparación con la primera. La reducción abierta y fijación interna de los componentes anteriores del anillo pélvico, por lo general, se realizan con placas $y$, más recientemente, se ha propuesto el empleo de tornillos canulados cruzados. ${ }^{9,10}$ Aun así, los fijadores externos pueden ser empleados como tratamiento estabilizador permanente mientras se garantice una reducción satisfactoria. ${ }^{11}$ 
En nuestro hospital, no se contaba con un estudio epidemiológico que permitiera identificar los mecanismos de lesión, edad y sexo más frecuentes de los pacientes con fractura de pelvis valorados por nuestro servicio, así como la mortalidad entre los mismos. El objetivo además de identificar estas características epidemiológicas, fue hacer una revisión de los protocolos de manejo ortopédico aplicados.

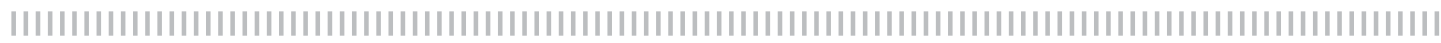

\section{Material y métodos}

Se realizó una búsqueda en la base de datos del expediente electrónico del nuestro hospital, con las palabras $<<$ fractura $>>$ $<<$ pelvis $>><<$ Tile $>>$. La búsqueda arrojó 39 pacientes, de los cuales sólo 28 se trataban de fracturas de pelvis valoradas por el Servicio de Traumatología y Ortopedia con expediente y protocolo radiográfico completos disponibles para su revisión, que correspondieron al periodo de septiembre 2014 a diciembre 2017.

También se realizó una búsqueda en la bitácora de ingresos y egresos al Área de Choque del Servicio de Urgencias, iden- tificando 12 defunciones (nueve del sexo masculino y tres del sexo femenino) que tenían el diagnóstico de politraumatismo, pero sin diagnóstico de fractura de pelvis clínica, por lo que no pudieron ser incluidos en esta serie.

De cada expediente, se incluyeron las siguientes variables: mes y año de la lesión, el sexo y edad del paciente, el mecanismo y ambiente de la lesión, el tratamiento quirúrgico de emergencia otorgado, las lesiones asociadas, el compromiso del aniIlo pélvico, las clasificaciones de Tile y AO otorgadas al ingreso, el tratamiento quirúrgico ortopédico definitivo y la mortalidad.

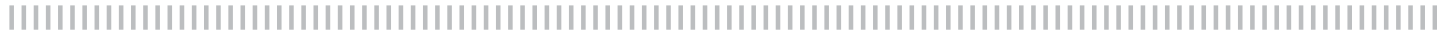

\section{Resultados}

Durante el periodo de septiembre 2014 a diciembre 2017, 28 pacientes del sector civil del Centenario Hospital Miguel Hidalgo $(\mathrm{CHMH})$, 19 masculinos $(67.8 \%)$ y 9 (32.1\%) femeninos, con una edad promedio de 32.7 años, con un rango entre 8 a 71 años de edad, con diagnóstico de fractura de pelvis, estables e inestables, de las cuales $32.1 \%(n=9)$ fue clasificado como Tile/AO A, 32.1\% $(n=9)$ como B y $35.7 \%(n=10)$ como $C$; desde septiembre a diciembre del año 2014 se registraron dos pacientes, seis durante 2015, diez en 2016 y diez en el año 2017.

En el $64.2 \%(n=18)$ de los casos revisados, la lesión fue por un mecanismo de accidente de vehículo motor, $3.5 \%(n=1)$ por caída de motocicleta, 3.5\% $(n=1)$ por caída del plano de sustentación, 10.7\% $(n=3)$ por caída mayor a un metro de al- tura, 7.14\% $(n=2)$ por caída de caballo y $10.74 \%(n=3)$ por atropellamiento. De éstos, el $64.2 \%(n=18)$ fue en un ambiente urbano, $14.2 \%(n=4)$ rural, $7.14 \%(n=2)$ autopista o carretera y $14.28 \%(n=4)$ en casa. Tras su admisión al servicio de urgencias, durante su valoración multidisciplinaria $42.8 \%(n=12)$ no requirió de algún procedimiento quirúrgico de urgencia, a $42.8 \%$ $(n=12)$ se le colocó un fijador externo de urgencia como tratamiento por inestabilidad pélvica, todos por parte del servicio de traumatología y ortopedia; del total de pacientes, a $14.28 \%(n=4)$ se le realizó laparotomía por parte del servicio de cirugía general y solo $7.14 \%(n=2)$ ameritó tratamiento con aseo quirúrgico por exposición de la fractura pélvica por parte de traumatología y ortopedia; pese a que $25 \%(n=7)$ de los pacientes no presentaba alguna le- 
sión asociada, $67.85 \%(n=19)$ presentaba otras fracturas además de la lesión pélvica, $14.28 \%(n=4)$ manifestó lesión urológica, diagnosticada por el servicio de cirugía general al momento del ingreso o durante su estancia hospitalaria; 3.5\% $(n=1)$ presentaba lesión de otros órganos abdominales y 3.5\% $(n=1)$ lesión vascular arterial asociada; ningún paciente presentaba lesión medular asociada.

Respecto al componente comprometido del anillo pélvico, en $3.5 \%(n=1)$ no se involucraba con los componentes del anillo pélvico; sin embargo, en el 32.14\% $(n=9)$ se afectaba el componente anterior de forma bilateral, en el $21.42 \%(n=6)$ el anillo anterior derecho y en el $17.85 \%(n=5)$ el izquierdo; en $7.14 \%(n=2)$ involucraba de forma bilateral los componentes posteriores (sacroilíacas), en $14.28 \%(n=4)$ el derecho y $14.28 \%(n=4)$ el izquierdo; en $21.42 \%$ $(n=6)$ se reportó diástasis de la sínfisis pubiana. Nueve pacientes presentaron compromiso óseo de los componentes anterior y posterior del anillo pélvico. Un $42.85 \%$ $(n=12)$ de los pacientes no ameritó tratamiento quirúrgico. $17.85 \%(n=5)$ continuó con fijador externo como tratamiento definitivo; a un $25 \%(n=7)$ se le realizó reducción abierta y fijación interna con placas, $10.71 \%(n=3)$ se fijó con una combinación de placas y tornillos sacroilíacos, y a un $3.5 \%(n=1)$ solo con tornillo sacroilíaco.

Se registraron dos defunciones (7.14\%) $y$ dos pacientes fueron trasladados a otro hospital; el $85.71 \%(n=24)$ de los pacientes egresó por mejoría del hospital.

TRATAMIENTO ORTOPÉDICO DEFINITIVO

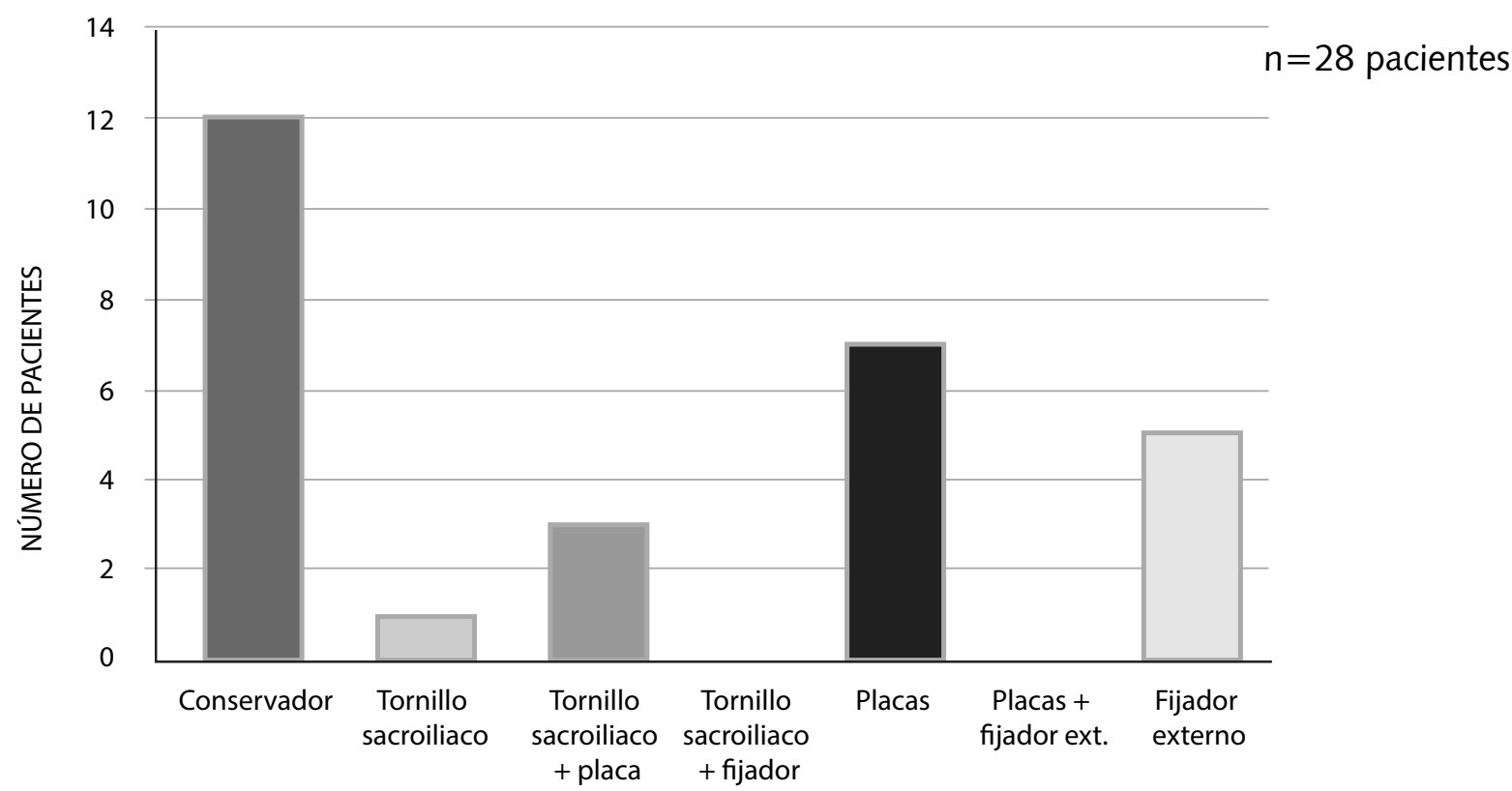

OSTEOSÍNTESIS DEFINITIVA

Figura 1. Distribución nominal de acuerdo con fijación ortopédica definitiva. 


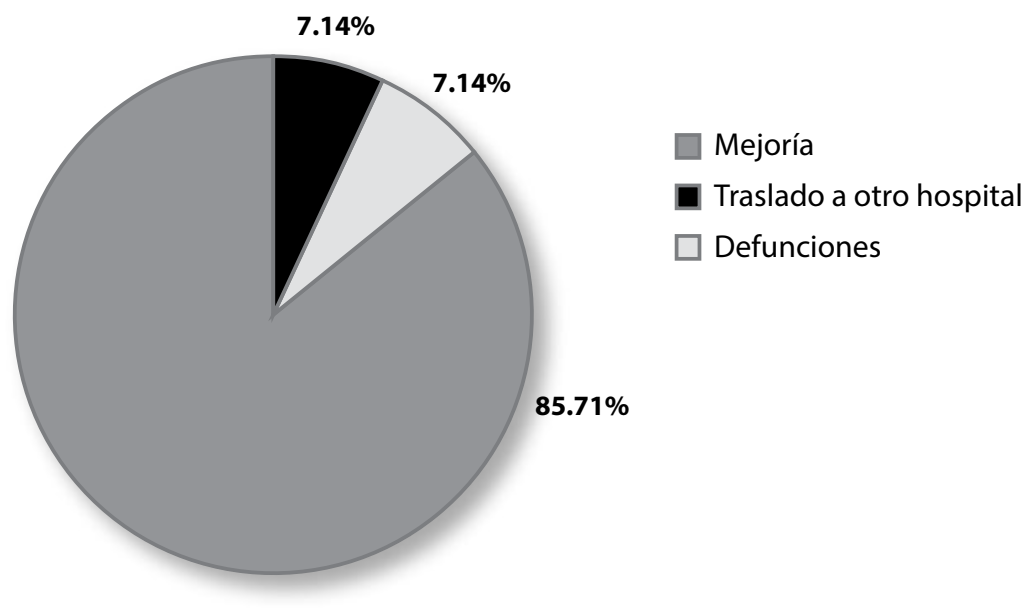

Figura 2. Motivos de egreso de los pacientes tratados por fracturas de pelvis.

\section{Discusión}

Las fracturas de pelvis inestables han ido en aumento progresivo anualmente. La edad promedio reportada en esta serie es de 32.7 años, con predominio del sexo masculino en áreas urbanas y secundarias a mecanismos de alta energía por accidentes de tráfico; no tan distinto a lo reportado por Cacão GJ et al. ${ }^{12}$ quien reportó 66 pacientes con edad media de 47 años, en su mayoría masculinos de raza blanca con fracturas Tile A.

La mayor parte de las fracturas fueron clasificadas como estables; aquellas clasificadas como inestables en el grupo $\mathrm{C}$ de Tile/AO recibieron todas $(n=9)$ una fijación externa urgente en área de choque o quirófano así mismo tres pacientes clasificados como Tile $B$, en todos independientemente de la necesidad de otro procedimiento quirúrgico urgente; Hermans $E$ et al. ${ }^{13}$ revisaron una serie de 537 pacientes en quienes se reportó una fijación urgente con reducción abierta y fijación interna (RAFI) en un paciente con fractura Tile $A$, 41 pacientes de grupo $B$ con RAFI y 15 con fijador externo; en pacientes Tile $C$, 15 pacientes con fijador externo, 7 con clamp-C y 96 pacientes con RAFI urgente; esto supone una intervención con impacto significativo en la reducción de la mortalidad de $7.14 \%$ en pacientes atendidos en el $\mathrm{CHMH}$, comparándose con la reportada por Hermans en Holanda de $13.6 \%$, y por encima de la reportada por Cacão en Brasil de 3\%. En un análisis retrospectivo de 179 pacientes, Kheong et al. ${ }^{14}$ reportaron la severidad de la lesión pélvica, choque o coma a la admisión, y trauma craneoencefálico y torácico como factores predictores de mayor mortalidad. ${ }^{14}$

Es válido cuestionar si la mortalidad arrojada en el presente trabajo resulta realmente influenciada por la fijación externa en paciente con inestabilidad pélvica desde su ingreso; así mismo, debido a la naturaleza de las lesiones propias y asociadas, es común que gran parte de los pacientes con fracturas pélvicas fallezcan en el sitio del traumatismo o durante su valoración inicial en urgencias, por lo que es difícil establecer una cifra de mortalidad global. 
Aunque una reducción satisfactoria permite continuar con la fijación externa como osteosíntesis definitiva, solo en el 31.25\% de los pacientes en quienes se decidió tratamiento quirúrgico, continuaron con esta modalidad de tratamiento, en la mayoría se prefiere establecer fijación interna para evitar las potenciales complicaciones tradicionalmente descritas. Sin embargo como se revisó por Tosounidis et al, en 59 pa- cientes estas complicaciones además de bajas en incidencia, no afectan el resultado funcional final.

En su mayoría, la fijación interna se obtuvo con placas $(43.75 \%)$, todas de reconstrucción y en $18.75 \%$ una combinación de tornillos canulados sacroilíacos y placa anterior; lo que se traduce en una mayoritaria experiencia quirúrgica en los abordajes pélvicos.

\section{Conclusiones}

- En nuestro hospital la colocación inmediata de fijadores externos se realiza de forma oportuna tras la identificación de inestabilidad pélvica.

- Se prefiere la osteosíntesis definitiva con fijación interna tras la estabilización inicial.

- Reportamos una mortalidad aceptable respecto a otras series, aunque no puede atribuirse solamente al empleo urgente de fijadores externos.

\section{Bibliografía}

1. Miranda Roa JA, Hernández Manzo Jl. Estudio epidemiológico de los pacientes con fractura de pelvis en el servicio de reanimación del hospital general de Balbuena. Acta Ortop Mex 2006, 20(6): 256-261.

2. INEGI. Estadísticas de accidentes de tránsito terrestre en zonas urbanas y suburbanas. Disponible en http://www.inegi.org.mx/saladeprensa/aproposito/2016/trafico2016_0.pdf

3. Guthrie HC, Owens RW, Bircher MD. Fractures of the pelvis. J Bone Joint Surg 2010; 92-B: 1481-8.

4. Langford JR, Burgess AW, Liporace FA, Haidukewych GJ. Pelvic fractures: part 1. Evaluation, classification, and resuscitation. J Am Acad Orthop Surg 2013; 21: 448-457

5. Mella-Schmidt Claudio, Núñez-Contreras Álvaro. Clasificación de las fracturas de pelvis. Acta Ortop Mex 2008;4(4):234-241.

6. Vardon F, Brunel E. External contention for pelvic trauma: is 1 sheet enough? Am J Emerg Med. 2013; 31, 442,e1-442.e3

7. Yang $A P$, lannacone WM. External fixation for pelvic ring disruptions. Ortop Clin North America 1997;28(3):

8. Ronghe G, Huang W, Yang L, Liu H, Xie K, Huang Z. Comparison of front plate, percutaneus sacroiliac screws, and sacroiliac anterior papilionaceous pla- te in fixation of unstable pelvic fractures. Medicine 2017: 96:36

9. Yao F, He Y, Qian H, Zhou D, Li Q. Comparison of biomechanical characteristics and pelvic ring stability using different fixation methods to treat pubic symphysis diastasis. Medicine. 2015;94(49): e2207. doi: 10.1097/MD.0000000000002207

10. Gonzalvez AL, Martinez J, Cano P, Jimenez J, Sueiro J, Giraldez MA. Is cannulated-screw fixation an altervative top late osteosynthesis in open book fractures? A biomechanical analysis. Injury, Int. J Care Injured 2016;47S3: S72-S77

11. Tosounidis TH, Sheikh HQ, Kanakaris NK, Giannoudis PV. The use of external fixators in the definitve stabilisation of the pelvis in polytrauma patientes: Safety, efficacy and clinical outcomes. Injury, Int, J, Care Injured 2017; S0020-1383(17)30185-7.

12. Cacão GJ, Ribeiro $E$, Innocenti $D$, Marques F, Bartelega J, da Costa B. Epidemiology of pelvic ring fractures and injuries. Rev Bras Ortop. 2017; 52(3): 260-269.

13. Hermans E, Biert J, Edwards MJR. Epidemiology of pelvic ring fractures in level 1 trauma center in the netherlands. Hip Pelvis 2017; 29(4): 253-261.

14. Kheong C, Goh HK, Tay SY. Patients with pelvic fracture: what factors are associated with mortality? Int J Emerg Med (2010) 3:299-304. 